difficulté réside surtout dans l'agencement optimal des différents éléments pour l'esthétique et la facilité de montage.

\title{
7.3 Position de la France
}

Pour des raisons historiques et culturelles, la France s'est spécialisée dans la pierre, la brique et le béton. Les métaux n'y ont qu'une place modérée ( $c^{\prime}$ est aussi le cas du bois, sauf en montagne). L'image tenace de mauvaise tenue au feu des structures en acier lors de la catastrophe du CES Pailleron, il y a presque 40 ans, y est pour quelque chose. Depuis, des solutions fiables ont été développées mais l'image persiste. Ailleurs (Angleterre, Japon), on a fait des choix différents avec une forte participation du métal dans la construction. Les centres de production et de recherche sont donc plutôt à l'étranger : Angleterre, Finlande, Japon, Belgique et Luxembourg.

- Un effort de recherche et de développement tout particulier doit être fait pour améliorer cette situation. Ceci est d'autant plus vrai que la France dispose d'un centre de la construction métallique (CTICM) qui rassemble les compétences en la matière.

\section{Métaux et Défense}

Les matériaux utilisés par la Défense sont soumis à des sollicitations extrêmes (chargement, vitesse de déformation, température) et doivent permettre des durées $d^{\prime}$ utilisation très longues (corrosion) (annexe 16). Le comportement d'un blindage soumis à un impact de projectile dépend de façon notable des caractéristiques dynamiques de l'impact lui-même et la compréhension des phénomènes aux grandes vitesses de déformation - en particulier changements de phase ou transformations internes - est encore imparfaite.

On ne saurait oublier la supériorité qu'a longtemps donnée, aux combattants, la maîtrise d'une bonne Métallurgie : que l'on songe seulement aux épées damassées des Arabes au temps des croisades!

En France, Métallurgie et Défense ont une vieille histoire en commun, de recherche et d'innovations, en particulier au Laboratoire Central de la Marine où des progrès décisifs ont été réalisés, dans les années 1950, sur la technique du polissage électrolytique des métaux. 


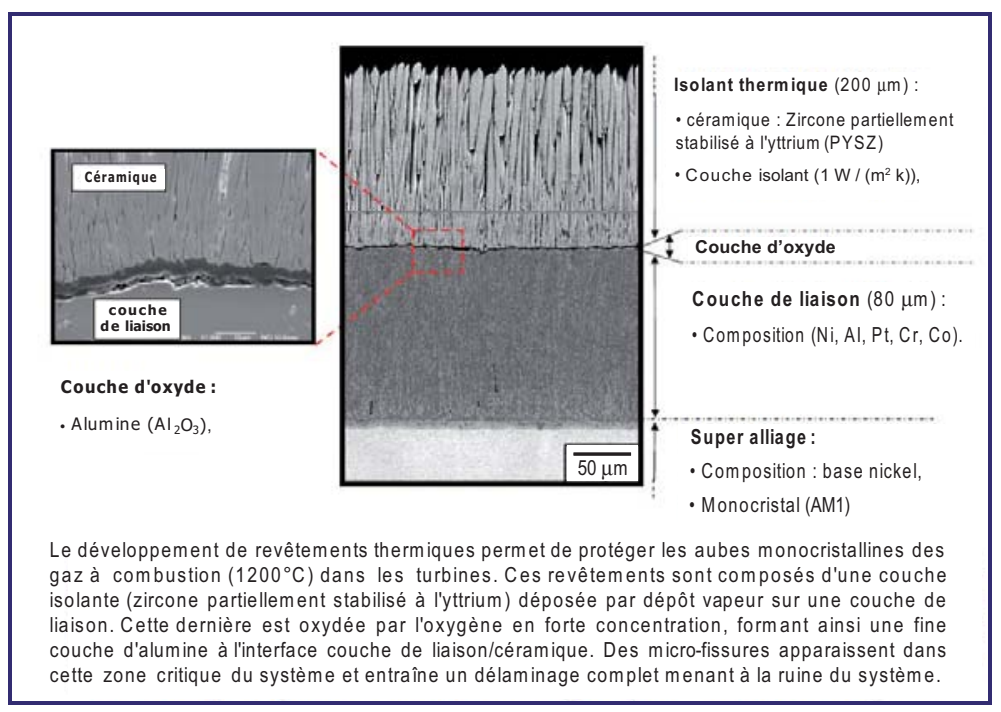

\section{Figure 2.5}

Aube monocristalline du moteur M88 de l'avion de combat Rafale. Les aubes sont isolées thermiquement à l'aide d'un dépôt de zircone (cf. figure 2.1, chapitre 2) (Thèse de Julien Frachon, ENSMP, 2009).

La Défense a besoin de matériaux métalliques aux propriétés supérieures à celles des applications civiles : tenue à températures élevées (matériaux moteurs, voir figure 2.5), caractéristiques mécaniques extrêmes (tenue à l'impact, perforation blindage), module élevé et très haute résistance, tenue à l'écrasement des coques de sous-marins, comportement à très long terme maîtrisé et garanti, en plus du problème de la sécurisation des sources d'approvisionnement.

L'effort de recherche soutenu par la Défense, et à maintenir, doit porter principalement dans les domaines suivants :

- amélioration des techniques de mise en œuvre (recherche d'une faible dispersion des caractéristiques, contrôle des microstructures, assemblage par soudage);

- renforcement de la tenue au vieillissement sur de très longues durées et dans des environnements très sévères (température, humidité);

- mise en place de nouvelles technologies de traitements de surface, respectant le règlement $\mathrm{REACH}^{6}$;

- meilleure connaissance du comportement dynamique des matériaux métalliques. Dans ce domaine, la DGA a fortement soutenu la création de

\footnotetext{
${ }^{6} \mathrm{REACH}$ : Registration, Evaluation, Autorisation and Restriction of Chemicals.
} 
I'association DYMAT ${ }^{7}$ il y a plus de vingt ans, et ne cesse de promouvoir l'intérêt de cette thématique auprès de la communauté scientifique concernée. Cet appui doit être maintenu, voire renforcé.

\section{9 | Biomatériaux métalliques}

On appelle biomatériaux « des matériaux non vivants utilisés dans un dispositif médical destiné à interagir avec les systèmes biologiques ». En contact avec la matière vivante (implants...), ils mettent en jeu la biocompatibilité et la bio-activité. Ce sont souvent des céramiques, des polymères, des matériaux $d^{\prime}$ origine naturelle mais nous n'évoquerons ici que le cas des métaux et alliages (annexe 17).

Parmi ces derniers, les plus utilisés actuellement sont :

- les aciers inoxydables $316 \mathrm{~L}$ pour les stents*, les vis et plaques de fixation, les valves...;

- les alliages de titane (prothèses de hanche, vis, implants dentaires) présentant une excellente résistance à la corrosion, une faible densité, une bonne biocompatibilité (I'os adhère facilement au titane) mais une faible résistance à l'abrasion;

- les alliages à mémoire de forme type nitinol, Ti-55 \% Ni (stents, odontologie...);

- les ailiages cobalt-chrome (odontologie, prothèses de hanche et de genou, valves cardiaques) présentant une grande résistance à l'usure.

Mais nombre de problèmes subsistent en particulier l'émission dans l'organisme d'ions métalliques (nickel, cobalt) classés cancérigènes, ou la création de débris par frottement.

Il convient donc de mettre au point de nouveaux alliages sans nickel ni cobalt, résistants à la corrosion et ayant de bonnes propriétés mécaniques (par exemple des aciers austénitiques sans nickel) ; d'autre part d'étudier des revêtements polymères ou céramiques - par exemple carbure de silicium, oxyde ou nitrure de titane, zircone, carbone pyrolytique... - conférant à l'alliage une bonne résistance à l'usure et à la corrosion et permettant de limiter les frottements. Nombre $d^{\prime}$ alliages sont actuellement étudiés : alliages de cobalt pour l'arthroplastie du genou, alliages de titane de faibles modules d'Young pour leur biocompatibilité mécanique avec les os...

\footnotetext{
${ }^{7}$ DYMAT : European association for the promotion of research into the dynamic behaviour of materials and its applications.
} 